\title{
Supplementary Material for Inference for Respondent-Driven Sampling with Misclassification
}

\section{Supplement A: Performance of the Analytical Adjustment with the Salganik-Heckathorn Estimator}

We discuss here why the $c$-factor and its observed version $c^{*}$ both play a role in whether or not the linear adjustment applies to the Salganik-Heckathorn estimator.

If $\hat{\mu}_{S H}^{a d j}$ was successful at eliminating misclassification error, then we would have that

$$
\hat{\mu}_{S H}^{a d j}-\hat{\mu}_{S H}=0 .
$$

Since we have, by definition, that:

$$
\begin{aligned}
\hat{\mu}_{S H}^{\text {naive }} & =\frac{\hat{\mu}_{V H}^{\text {naive }}}{\hat{\mu}_{V H}^{\text {naive }}+c^{*}\left(1-\hat{\mu}_{V H}^{\text {naive }}\right)} \\
\therefore \hat{\mu}_{S H}^{\text {adj }} & =\frac{\hat{\mu}_{V H}^{\text {naive }}-f^{+}\left(\hat{\mu}_{V H}^{\text {naive }}+c^{*}\left(1-\hat{\mu}_{V H}^{\text {naive }}\right)\right)}{\left(1-f^{+}-f^{-}\right)\left(\hat{\mu}_{V H}^{\text {naive }}+c^{*}\left(1-\hat{\mu}_{V H}^{\text {naive }}\right)\right)},
\end{aligned}
$$

and in addition, since $\hat{\mu}_{V H}^{a d j}-\hat{\mu}_{V H} \approx 0$, we have that:

$$
\begin{aligned}
\hat{\mu}_{S H}=\frac{\hat{\mu}_{V H}}{\hat{\mu}_{V H}+c\left(1-\hat{\mu}_{V H}\right)} & \approx \frac{\hat{\mu}_{V H}^{\text {adj }}}{\hat{\mu}_{V H}^{a d j}+c\left(1-\hat{\mu}_{V H}^{\text {adj }}\right)} \\
& =\frac{\hat{\mu}_{V H}^{\text {aive }}-f^{+}}{\left(1-f^{+}-f^{-}\right)+(c-1)\left(1-f^{-}-\hat{\mu}_{V H}^{\text {naive }}\right)},
\end{aligned}
$$

and therefore, we may conclude that:

$$
\begin{aligned}
\hat{\mu}_{S H}^{a d j}-\hat{\mu}_{S H} \approx & {\left[\frac{\hat{\mu}_{V H}^{\text {naive }}-f^{+}\left(\hat{\mu}_{V H}^{\text {naive }}+c^{*}\left(1-\hat{\mu}_{V H}^{\text {naive }}\right)\right)}{\left(1-f^{+}-f^{-}\right)\left(\hat{\mu}_{V H}^{\text {aive }}+c^{*}\left(1-\hat{\mu}_{V H}^{\text {naive }}\right)\right)}\right]-} \\
& {\left[\frac{\hat{\mu}_{V H}^{\text {naive }}-f^{+}}{\left(1-f^{+}-f^{-}\right)+(c-1)\left(1-f^{-}-\hat{\mu}_{V H}^{\text {naive }}\right)}\right] . }
\end{aligned}
$$

Therefore, the right hand side of this equation equals zero, for $c=c^{*}=1$. However, other values for $c$ and/or $c^{*}$ may result in $\hat{\mu}_{S H}^{a d j}-\hat{\mu}_{S H}$ being different than zero, thus indicating that the analytical adjustment will have a poor performance. This has been found to indeed create biases in the simulations. 
In practice, it is not possible to calculate $c$ in presence of misclassification. Although no exact linear relationship exists between $c$ and $c^{*}$, as seen in Figure from our simulations, they tend to be positively correlated. As such, a high $c^{*}$ may imply an elevated $c$ and should serve as an indicator that the analytical adjustment might not be the best correction method for the Salganik-Heckathorn estimator.
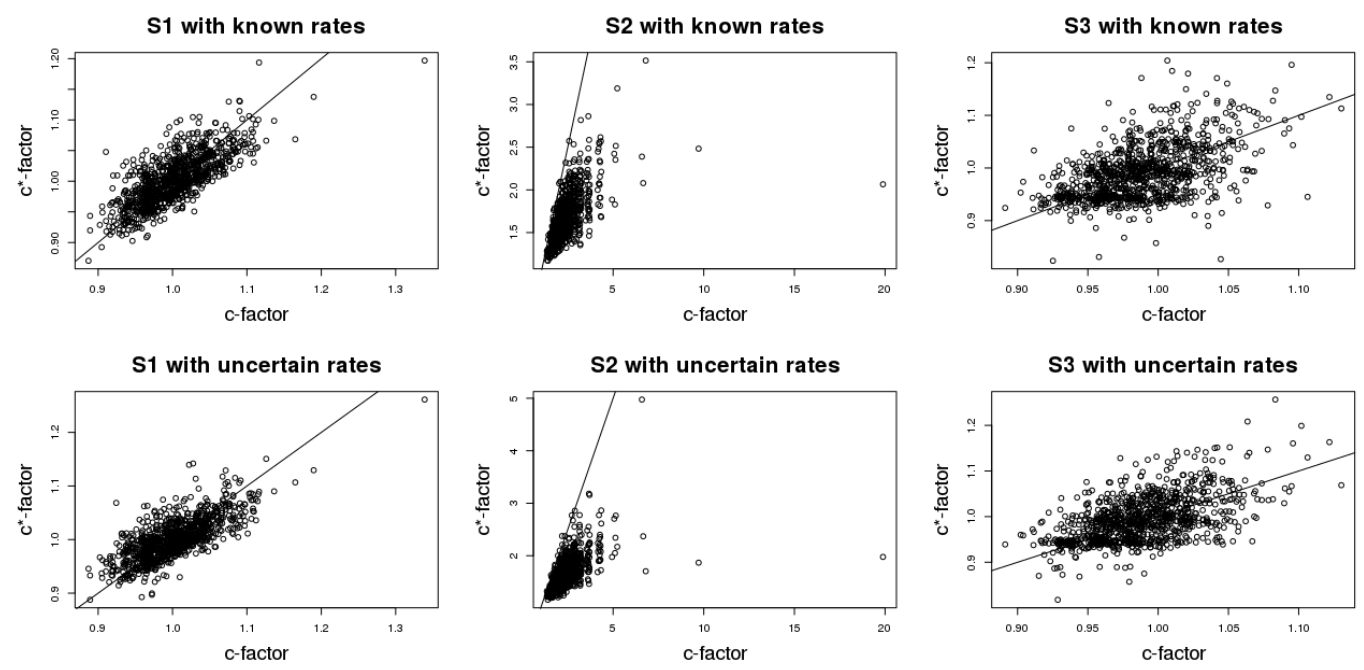

Figure 1: Relation between $c^{*}$ and $c$ under the simulation study scenarios.

\section{Supplement B: Additional Results From Simulation Study}

\section{Root Mean-Squared-Error}

Table 1 shows the components of the Mean-Squared-Error as well as the Root meansquared-error (RMSE). Discussion about these results may be found in Section 4.2.

\section{RMSE at Various Levels of Misclassification Rates}

Figure 2 presents the average RMSE improvement when using the analytical adjustment compared to the naive approach, that is:

$$
\frac{\overline{R M S E}_{\text {naive }}-\overline{R M S E}_{\text {adj }}}{\overline{R M S E}_{\text {naive }}} .
$$

The calculations were performed with false positive and negative rates varying from 0 to 0.4 by 0.04 increments and under scenario 1 for the Volz-Heckathorn estimator. The average improvement is expressed as a function of the average misclassification error present in the estimates. In most instances, the RMSE is significantly lower than under the naive approach. The limited instances where the average RMSE with the analytical adjustment is higher than the naive one occur when the estimates 


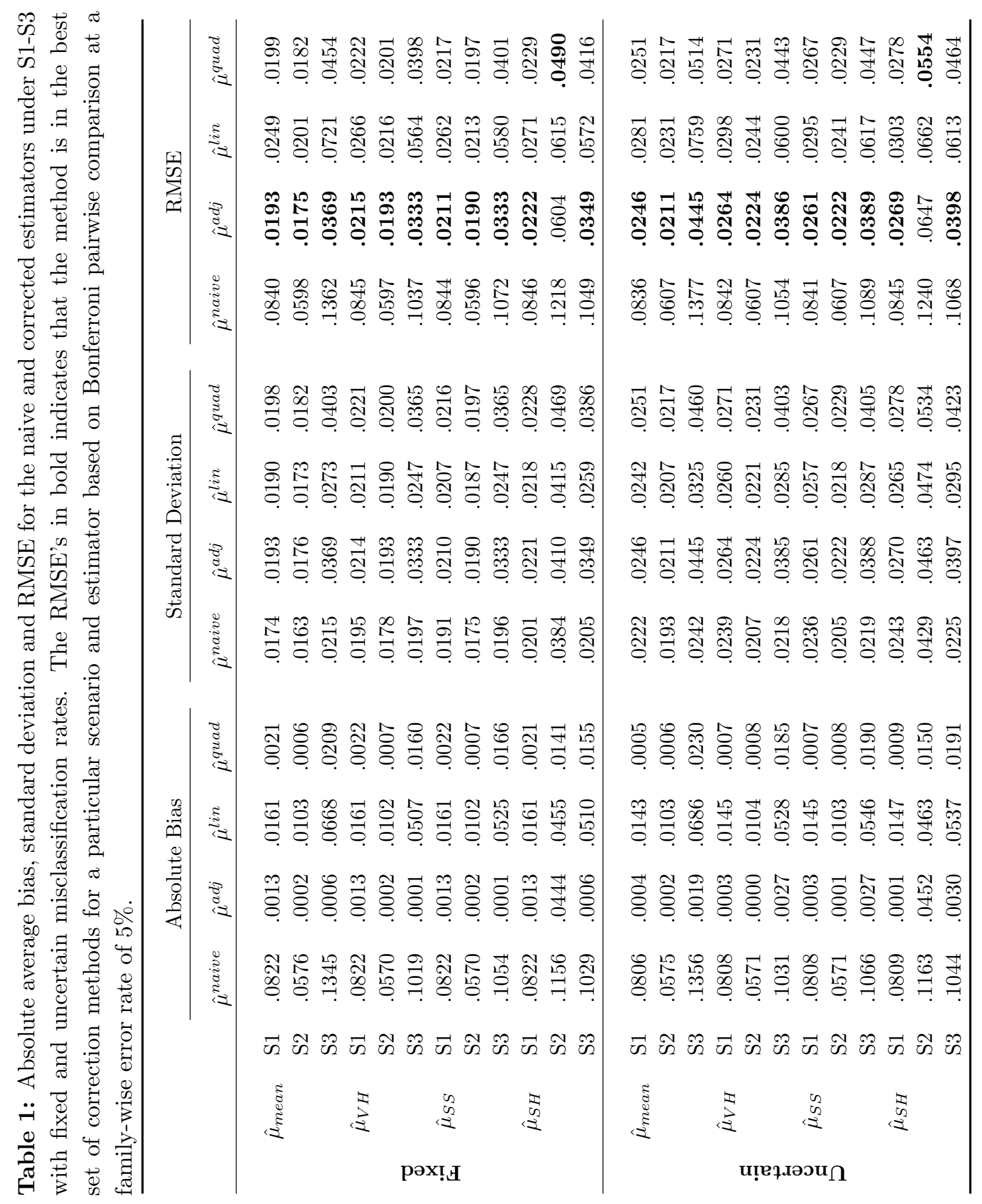


contain little misclassification error. In those cases, the benefits from the reduction in misclassification error are offset by the increase in the uncertainty of the estimates.

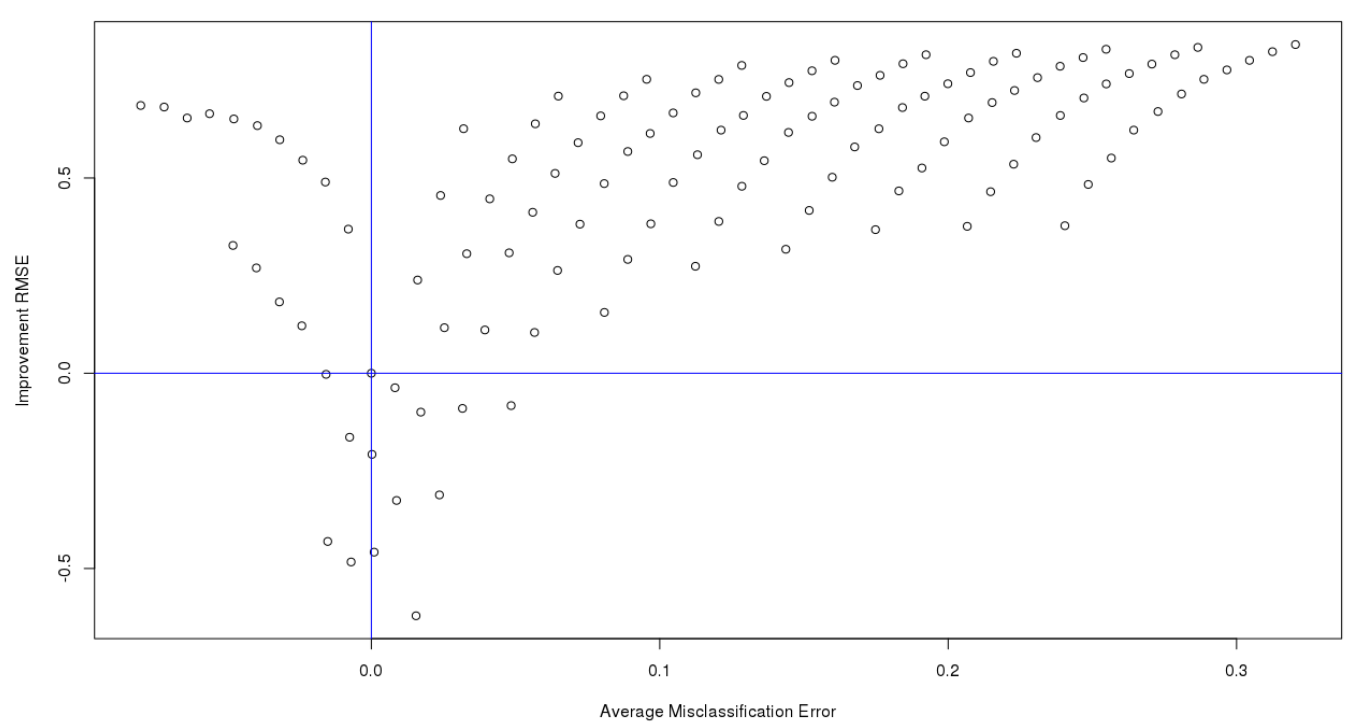

Figure 2: Relative decrease in the average RMSE for the Volz-Heckathorn estimator under $\mathrm{S} 1$ as a function of the average misclassification error in the estimates.

\section{Sensitivity to Erroneous Error Rates}

Figure 3 shows the misclassification error still present in the Volz-Heckathorn estimates after applying the analytical adjustment when inaccurate misclassification error rates (i.e. $f^{+}$and $f^{-}$) are used in equation (3.2). The impact of inaccurate rates is presented for $\mathrm{S} 1$ to $\mathrm{S} 3$ at various levels of inaccuracy in $f^{+}$and $f^{-}$. The relation is shown in terms of $f^{+}$for $\mathrm{S} 1$ and $\mathrm{S} 2$ and $f^{-}$for S3 since those rates significantly deviate from the true rates under the corresponding scenarios. As for the dash line, it represents the average misclassification error in the naive point estimate. Very few point estimates in either of the three scenarios contain more misclassification error than the one present in the average naive point estimate. This suggests that for moderate departure from the true misclassification rates, the correction methods may still result in less misclassification error than the naive approach. Although Figure 3 is based on inaccurate $f^{+}$and $f^{-}$for all scenarios, the uncertain $f^{-}$'s in S1 and S2 and $f^{+}$'s in S3 are fairly close to the true rate. 

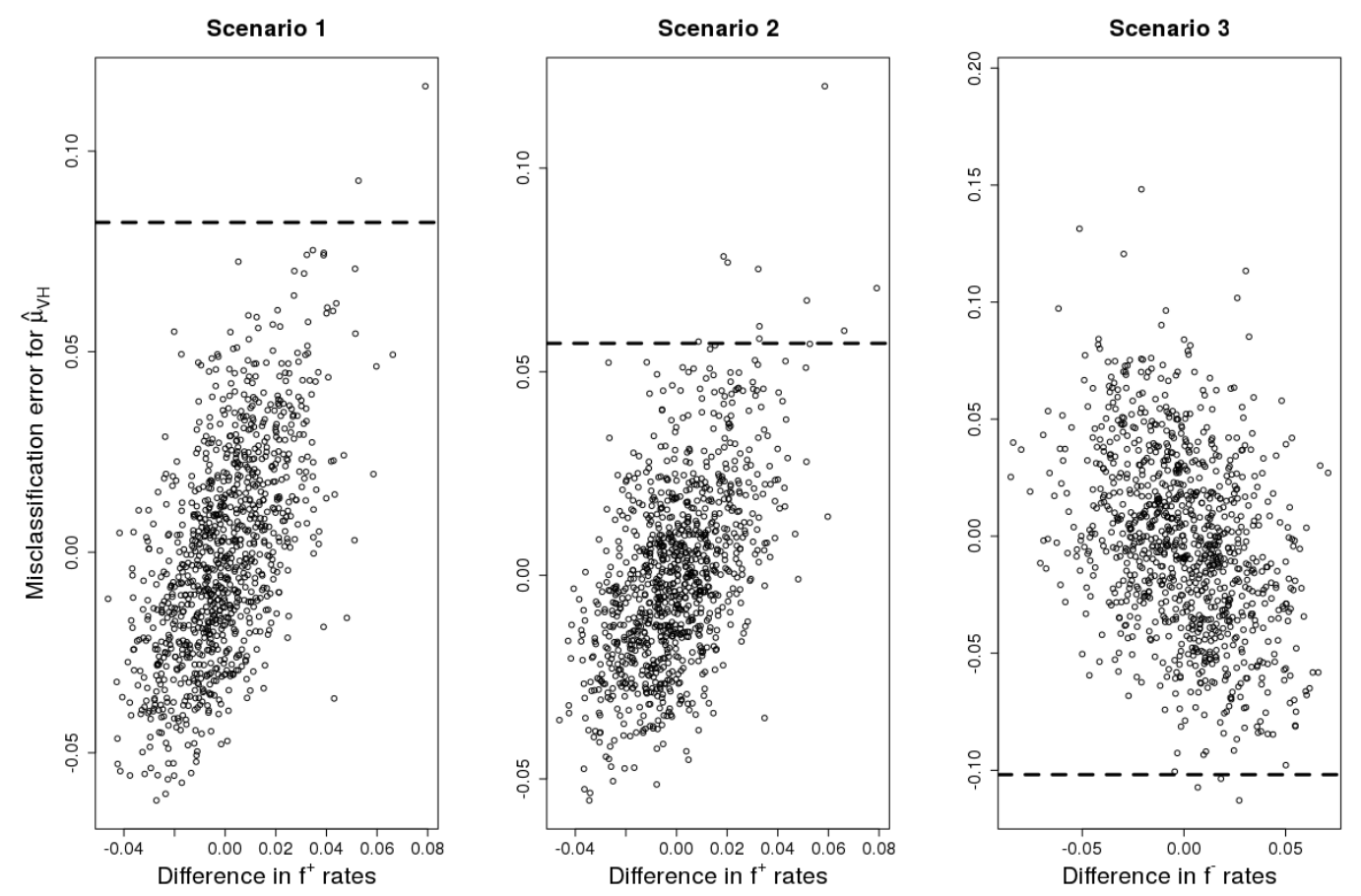

Figure 3: Misclassification error remaining in the VH estimates $\left(\hat{\mu}_{V H}\right)$ after applying the analytical adjustment for S1 to S3 as a function of the inaccuracy in the error rates (either $f^{+}$or $f^{-}$). The dash line represents the average misclassification error in the naive point estimate. 